\title{
Une exploration interdisciplinaire des liens entre relation au lieu et concernement. À propos des risques fluviaux et côtiers en France métropolitaine
}

François Bousquet ${ }^{1, *}$ (D), Nicolas Rocle ${ }^{2}$ (D), Hélène Rey-Valette ${ }^{3}$, Catherine Meur-Ferec ${ }^{4}$ (D), Didier Vye ${ }^{5}$, Nicole Lautrédou-Audouy ${ }^{6}$, Marion Amalric ${ }^{7}$ (D), Lucile Blanchet ${ }^{8}$ (D), Sandrine Lyser ${ }^{9}$ (D), Caroline Blondy ${ }^{10}$ (D) et Nicolas Becu ${ }^{11}$ (D)

${ }^{1}$ Sciences de l'environnement, UMR SENS, CIRAD, IRD, Université Paul-Valéry-Montpellier-3, Université de Montpellier, Montpellier, France

${ }^{2}$ Sociologie, INRAE, UR ETBX, Cestas, France

3 Économie, CEE-M, Université de Montpellier, CNRS, INRAE, InstitutAgro, Montpellier, France

${ }^{4}$ Géographie, Université de Brest, Institut universitaire européen de la mer, UMR LETG, Brest, France

${ }^{5}$ Géographie, La Rochelle Université, UMR LIENSs, La Rochelle, France

${ }^{6}$ Sciences de l'environnement, CEE-M, Université de Montpellier, CNRS, INRAE, InstitutAgro, Montpellier, France

${ }^{7}$ Géographie, Université de Tours, UMR CITERES, Tours, France

${ }^{8}$ Géographie, Sorbonne Université, Paris, France

${ }^{9}$ Statistique, INRAE, UR ETBX, Cestas, France

${ }^{10}$ Géographie, La Rochelle Université, UMR LIENSs, La Rochelle, France

${ }^{11}$ Géographie, CNRS, UMR LIENSs, La Rochelle, France

Reçu le 5 avril 2019. Accepté le 25 mai 2020

Cet article propose une synthèse ex post de cinq enquêtes sur la perception des risques littoraux (submersion, inondation, érosion) par les personnes qui fréquentent ces lieux. Cette analyse a associé les auteurs des enquêtes de "première main » pour coconstruire la montée en généralité. En complément des approches sur la construction collective des territoires et des risques et leur mise en politique qui a fait l'objet de plusieurs articles dans la revue, cette contribution étudie les ressorts individuels du déni, de la mise à distance, de la vigilance et de l'engagement. Cette focale individuelle permet de comprendre des relations différentes, entre risque pour soi et risque subi par le collectif, selon la relation personnelle au lieu.

La Rédaction

\begin{abstract}
Résumé - Quels sont les liens entre la relation au lieu que développent des individus et leur «concernement» à propos de phénomènes qualifiés de «risques» sur ce même lieu? Afin de répondre à cette question, un état de l'art précise tout d'abord le concept de «concernement» en qualifiant différentes modalités (notamment «passif» ou «actif») ainsi que le concept de «relation au lieu» et ses différentes dimensions (identitaire, émotionnelle, symbolique, fonctionnelle). Sur la base de ces précisions, et à partir des données issues de différentes enquêtes menées sur les littoraux français, une analyse est ensuite menée pour explorer empiriquement les liens entre différentes dimensions de la relation au lieu et les modalités de concernement vis-à-vis des risques fluviaux et côtiers. Les résultats montrent des liens négatifs entre les dimensions symboliques et émotionnelles de la relation au lieu et un concernement qualifié de passif, ainsi que des liens positifs entre la relation symbolique au lieu et un concernement dit actif. Ces relations sont enfin discutées par rapport aux implications de ce type de résultats dans des processus de gouvernance.
\end{abstract}

Mots-clés : territoire / risque / concernement environnemental / relation au lieu / attachement

\footnotetext{
*Auteur correspondant : francois.bousquet@cirad.fr
} 


\begin{abstract}
Interdisciplinary exploration of the links between sense of place and individual concern. The case of river and coastal risks in metropolitan France. What are the links between the 'sense of place' of individuals and their 'concern' about coastal phenomena described as 'riskgenerating' on French coasts? A state of the art specifies the concept of 'sense of place' and its several dimensions (identity, emotional, symbolic, functional) as well as the notion of 'concern' and its modalities (passive or active). Based on these clarifications, as well as on data from a range of surveys conducted on French coastal and estuarine areas, we empirically characterize the dimensions of sense of place and their links with the modes of environmental concern. The results show negative links between the symbolic and emotional dimensions of relation to place and a concern qualified as passive, as well as positive links between the symbolic dimension of relation to place and a so-called active concern. We discuss these relationships as well as their consequences for coastal and territorial risk governance.
\end{abstract}

Keywords: territory / risk / environmental concern / sense of place / place attachment

Les zones côtières évoluent rapidement sous l'effet de nombreux processus écologiques, climatiques, sociaux, économiques, démographiques. Dans la perspective du changement climatique et de la montée du niveau de la mer, elles constituent des terrains d'observation privilégiés pour étudier les processus d'adaptation des sociétés, en particulier selon la relation des habitants vis-à-vis des risques d'érosion et de submersion. Cette relation est néanmoins complexe et les modèles classiques de rationalité fondés sur l'information et la réaction des agents à des incitations et à des contraintes (réglementaires ou financières) peinent à l'expliquer. Par ailleurs, les psychologues de l'environnement, les géographes et les sociologues ont développé différents concepts permettant de comprendre les comportements et les attitudes vis-à-vis du risque (Burley et al., 2007; Lewicka, 2011; Willox et al., 2012; Masten et Cicchetti, 2016). Parmi ces travaux, les recherches relatives au «sense of place», qui sera traduit ici par «relation au lieu» (qu'il ne faut pas confondre avec les recherches sur les lieux ou sur les territoires), apportent un regard complémentaire pour comprendre comment le risque est construit. Ces recherches posent aussi la question du lien entre « la relation au lieu » et les mobilisations individuelles ou collectives pour faire face au risque (Wester-Herber, 2004 ; Billig, 2006 ; Bernardo, 2013; Hellequin et al., 2013 ; De Dominicis et al., 2015 ; Rey-Valette et al., 2015; Quinn et al., 2019). L'objectif de cet article est d'analyser si, et selon quelles dimensions, la relation au lieu permet d'appréhender et d'expliquer différents types de «concernement» des populations face aux risques littoraux, la notion de « concernement» étant ici entendue comme une «sensibilité relative à un milieu donné [...] qui s'exprime par un comportement plus ou moins actif» (Brunet, 2008).

L'article s'appuie sur un travail mené dans le cadre d'un projet de recherche interdisciplinaire (projet MASALA, 2017-2018, mission pour l'interdisciplinarité du CNRS) qui visait, à partir d'enquêtes conduites sur le littoral français ces dix dernières années, à confronter les grilles d'analyse et les résultats afin d'expliciter les formes de relation aux risques côtiers (submersion et érosion) et d'inondation, ainsi que leurs impacts sur les comportements face à ces risques. Les questions posées étaient les suivantes: dans quelle mesure la relation au lieu, prise selon ses différentes dimensions, influence-telle la relation d'un individu à un phénomène qualifié de «risqué» sur les littoraux français? Quels sont les liens observés entre des types de «relation au lieu» et des degrés de «concernement» des populations par rapport aux risques littoraux et aux enjeux associés? Pour mener ce travail impliquant des géographes, des sociologues, des environnementalistes et des économistes, un état de l'art approfondi a été mené sur les notions de relation au lieu et de concernement, en montrant qu'elles nécessitent toutes deux d'être précisées car elles agrègent différentes dimensions: identitaire, émotionnelle, symbolique et fonctionnelle pour la relation au lieu, ainsi que des dimensions plus ou moins actives du concernement... Sur cette base, et à partir des données issues des différentes enquêtes, une réflexion interdisciplinaire a été menée pour caractériser empiriquement les relations au lieu et leurs interactions avec le concernement des populations face aux risques côtiers. Les disciplines représentées étaient l'économie, la géomorphologie, la géographie, la modélisation, la psychologie environnementale, les sciences de l'environnement, la science politique et la statistique (par ordre alphabétique).

Afin de rendre compte et de discuter des résultats de cette expérience de mise en commun d'enquêtes sur la relation au risque côtier, la première partie de l'article explore les apports de la littérature à propos de la notion de «concernement» et de la «relation au lieu». Dans un deuxième temps, la démarche méthodologique de confrontation des différents apports est exposée en présentant les principales caractéristiques des enquêtes initiales et les types de traitement réalisés pour caractériser les liens entre types de relations au lieu et types de concernement. Enfin, la troisième partie discute les principaux résultats au regard de la littérature et esquisse certaines implications pour la gouvernance territoriale des risques côtiers. 


\section{«Relation au lieu » et « concernement »: des relations à expliciter et à explorer}

\section{Le concernement}

Hormis en psychologie et en psychiatrie $^{1}$ ou dans quelques travaux en sociologie des mouvements sociaux et de l'engagement, la notion de «concernement»a jusqu'à présent été peu mobilisée en France. Le terme n'est pas, en effet, sans poser problème pour en retenir une acception partagée, dans la mesure où il n'a pas d'existence lexicale française car il est une transposition de l'expression anglaise «to feel concerned». De même qu'en psychologie environnementale (Schultz, 2001) [cf.infra], la dimension dynamique de l'expression mobilise à la fois un registre émotionnel (être inquiet pour) et renvoie à un répertoire d'actions (agir sur la cause qui nous concerne). L'emploi du verbe «to feel» traduit le fait que se sentir concerné implique de «ressentir» un lien avec une entité extérieure [...] (Mabi, 2014, p.96). Une autre étymologie possible converge tout de même avec la précédente: «La notion de concernement provient du terme «Betroffenheit» utilisé par les Allemands en sociologie des mouvements sociaux. [...] Se sent concernée, en effet, toute personne qui se sent affectée par des situations [...]» (Faburel et Tribout, 2011). Cela rejoint ainsi le fait que «[le] concernement est souvent d'abord induit par une rupture de ce que Giddens (1984) nomme la "sécurité ontologique", c'est-à-dire la confiance de base dans la fiabilité des personnes, des choses et des lieux qui nous entourent, et grâce à laquelle le cours de notre vie ordinaire peut se dérouler généralement sans problème » (Barbier et Nadaï, 2015). C'est en ce sens que Philippe Brunet, reprenant du Petit Robert la forme passive de ce lien («être concerné: être intéressé, être touché par quelque chose ») et empruntant la notion de «milieu local» de Canguilhem (1992), définit le concernement comme une "sensibilité relative à un milieu donné [...] qui s'exprime par un comportement plus ou moins actif » (Brunet, 2008). L'auteur plaide alors pour un «usage raisonné de la notion de "concernement" " afin d'analyser les modalités de veille, de vigilance et de mobilisations peu visibles d'individus et d'associations (contre l'industrie nucléaire dans le cas étudié).

Le concernement caractérise ainsi toute attention portée à une situation qui serait ou pourrait devenir problématique (comme la survenance d'un risque en zone côtière), sans pour autant donner lieu à des formes

\footnotetext{
${ }^{1}$ En psychologie, la notion de concernement a été introduite par Henri Grivois dans ses travaux sur la «psychose naissante» et caractérise l'expérience irrépressible d'un individu à se retrouver au centre des mouvements et des intentions du reste des hommes.
}

d'engagement et d'actions dans l'espace public ${ }^{2}$. En psychologie environnementale, le concernement est surtout étudié en rapport avec la protection de l'environnement naturel. D'après Fransson et Gärling (1999), le «concernement environnemental» fait référence à une attitude spécifique qui détermine les intentions ou plus généralement une attitude ou des valeurs vis-à-vis de l'environnement. Le concernement environnemental comprend une dimension cognitive et une dimension comportementale. Stern (2000) associe à la dimension cognitive les valeurs, la vision du monde et les attitudes. La dimension comportementale relève quant à elle de l'engagement direct de l'individu dans l'action pour l'environnement. Les auteurs font plus particulièrement référence à la théorie Valeur-Croyance-Norme (ValueBelief-Norm [VBN]) [Stern et Dietz, 1994]. En s'appuyant sur cette théorie, Schultz (2001) distingue des concernements environnementaux qualifiés d'égoïstes, d'altruistes et de biosphériques, et relie ces concernements aux valeurs (centrées sur soi, sur les autres ou sur la biosphère). Le concernement environnemental est souvent conceptualisé comme un point de vue sur le monde (worldview) faisant référence aux croyances d'un individu sur le monde dans lequel il vit (Dake, 1991). La méthode du New Environmental Paradigm (NEP) est souvent utilisée pour mesurer ce point de vue en estimant à quel point un individu perçoit une interconnexion entre les humains et la nature (Dunlap et Van Liere, 1978). Les recherches montrent qu'il existe une relation entre les dimensions cognitives et comportementales, démontrant que ceux qui ont un point de vue environnemental sur le monde ont plus de chance d'être impliqués dans des comportements pro-environnement (Stern et al., 1995 ; Dietz et al., 1998).

La recherche de points communs entre ces différentes visions du concernement par la psychologie environnementale et la sociologie fait ressortir une complémentarité à partir de la dimension cognitive et réflexive qui conditionne des attitudes et orientations face à des situations problématiques. La distinction se fait vis-à-vis des valeurs, des savoirs et des processus qui conditionnent ces attitudes, ce qui amènera dans la section méthodologie à construire différentes modalités du concernement (présence/absence, passif/actif) [Tab. 1]. Car l'intérêt de la notion de concernement pour aborder la question des risques environnementaux renvoie selon nous à au moins trois dimensions. Elle permet tout d'abord de prendre en compte, au-delà de la «construction

\footnotetext{
2 «Rester sensible, connectée, informée, investie, prête à, sans pour autant être active, telles peuvent être des modalités revendiquées d'une posture qui, si elle n'est pas mobilisée hic et nunc, reste intentionnellement mobilisable pour et par tout événement qui serait évalué comme moment critique de vérité [...]» (Brunet, 2008).
} 
Tab. 1. Les différentes formes de concernement.

\begin{tabular}{|l|l|l|}
\hline & Non-concernement & Concernement \\
\hline Passif & $\begin{array}{l}\text { Ignorer l'existence du phénomène et des risques } \\
\text { associés }\end{array}$ & $\begin{array}{l}\text { Connaissance/conscience du risque (échelle } \\
\text { habitat, commune...), expérience du risque, } \\
\text { inquiétude, vigilance passive (observation des } \\
\text { phénomènes), identification des dommages vécus/ } \\
\text { potentiels, etc. }\end{array}$ \\
\hline Actif & $\begin{array}{l}\text { Identification d'une situation problématique (pour } \\
\text { soi et/ou pour les autres) mais qui s'accompagne } \\
\text { d'une mise à distance (faire primer les aménités } \\
\text { sur les risques/menaces potentielles, compter sur } \\
\text { l'action collective, déléguer aux experts et aux } \\
\text { autorités publiques, etc.) }\end{array}$ & $\begin{array}{l}\text { État d'alerte, en quête de preuves (recherche } \\
\text { d'informations, recoupements entre observation et } \\
\text { informations scientifiques disponibles...), mise en } \\
\text { lien (discussion avec autrui, participation à des } \\
\text { réunions, etc.) }\end{array}$ \\
\hline
\end{tabular}

sociale » du risque (Duclos, 1987 ; Peretti-Watel, 2003 ; Hacking, 2008) et des intérêts des individus, leur expérience sensible avec les milieux :

«[...] le concernement arrive aux personnes et aux groupes sans qu'ils aient eu nécessairement besoin de construire préalablement leur intérêt dans un espace de calcul. On engage ici une dimension importante, souvent oubliée dans la sociologie des acteurs: celle des milieux associés. Des projets, des décisions, des conflits peuvent affecter des milieux ou placer des personnes ou des groupes en état d'alerte quant à leurs conséquences futures sur leurs milieux » (Chateauraynaud, 2007).

Ce faisant, l'attention se porte également sur l'importance accordée aux éléments physiques du lieu et aux «traces» matérielles qui peuvent constituer autant de «prises» pour le concernement (Stedman, 2003). La notion de concernement permet donc de rendre compte des relations économiques et sociales au risque et au lieu tout en ajoutant des dimensions plus «sensibles» et plus passives. L'usage de la notion de concernement permet également de tenir à distance le clivage entre risque objectivé et risque subjectif, encore trop souvent soustendu par les approches en termes de perceptions et de représentations sociales des risques, et de se départir ainsi d'une analyse en termes de «biais cognitifs » tel que le «biais d'optimisme». La notion de concernement réhabilite enfin les savoirs et les processus de réflexivité des individus : elle suppose en effet que ce rapport sensible s'établit à partir d'un état de connaissances à propos d'un lieu ou d'une situation donnée. Il y a donc toute raison d'explorer plus avant ce qui, dans la relation au lieu (et aux milieux «à risque »), pourrait jouer dans le concernement des populations face aux risques littoraux et fluviaux.

\section{La relation au lieu}

La relation au lieu est étudiée par plusieurs disciplines, notamment la géographie et la psychologie.
La relation entre des individus (ou des groupes sociaux) et l'espace dans lequel ils vivent est en effet une des questions fondamentales en géographie (Di Méo, 2004 ; Debarbieux, 2012) comme le montrent les notions de géographicité - «relation existant entre l'homme et l'espace» (Dardel, 1952) ou d'écoumène - «relation de l'humanité à l'étendue terrestre » (Berque, 2000). Parfois, l'espace se confond avec la nature et à ce titre, de nombreux géographes privilégient l'analyse du lien entre nature et société (Gold, 1980; Pinchemel et Pinchemel, 1997), d'abord à travers la notion de milieu, propre à l'école française de géographie puis, plus récemment, à travers celle d'environnement, d'origine anglophone, et de territoire. Parce que l'espace est constitué de lieux, les géographes ont défini très tôt leur discipline comme une "science des lieux», le lieu étant à la fois « la plus petite unité spatiale complexe de la société [...], une condition de l'expérience humaine [...] et un espace dans lequel la distance n'est pas pertinente» (Lévy et Lussault, 2003). Ainsi, la géographie culturelle ou comportementale développe la notion de topophilie - littéralement amour des lieux - c'est-à-dire « le lien affectif qui existe entre les individus et un lieu donné » (Bachelard, 1957 ; Tuan, 1961) selon une orientation plutôt écologique et cosmologique. Plus récemment, le concept d'habiter, influencé notamment par la philosophie (Heidegger, 1958), la sociologie urbaine (Lefebvre, 1974) et l'anthropologie (Radkowski, 2002), est devenu un concept phare chez beaucoup de géographes francophones en particulier dans les champs du tourisme et de la géographie urbaine (Paquot et al., 2007). Concept multidimensionnel, l'habiter renvoie à l'ensemble des relations aux lieux, qu'elles soient idéelles ou matérielles, en insistant sur la dimension individuelle de ce lien. Ce concept permet d'intégrer clairement la question des mobilités spatiales dans l'analyse de la relation au lieu, que l'approche philosophique avait tendance à négliger (Stock, 2007). Ainsi, «habiter» se distingue totalement de «se loger» en géographie 
(Mathieu, 2016). La notion d'habiter poly-topique (Stock, 2006) permet également de montrer que les individus s'inscrivent dans des espaces de vie combinant plusieurs lieux distincts qui n'ont pas nécessairement la même valeur ou le même sens (Sébastien, 2016). De plus, puisque chaque usager d'un lieu peut l'habiter, ce concept élargit le qualificatif d'habitant, usuellement réservé aux résidents permanents, à l'ensemble des acteurs qui, par leurs mobilités, fréquentent temporairement un lieu donné : touristes, résidents secondaires, excursionnistes, «navetteurs », etc. Cette approche élargie de l'habitant prend tout son sens dans l'analyse des risques sur des littoraux particulièrement «mis en tourisme».

D'évidents liens peuvent être tissés entre les travaux des géographes et ceux des psychologues. En effet, depuis de nombreuses années la psychologie environnementale s'est intéressée à la relation entre les individus et le lieu dans lequel ils vivent. Le concept général qui recouvre d'autres concepts plus spécialisés est celui de « sens du lieu» (sense of place). Il comprend à la fois des dimensions cognitives, affectives, symboliques et identitaires (Hay, 1998; Jørgensen et Stedman, 2001; Stedman, 2002; Jørgensen et Stedman, 2006). Chacune de ces dimensions est étudiée à travers des concepts précis (Jørgensen et Stedman, 2001; Jørgensen et Stedman, 2006; Lewicka, 2011): place attachment (aspects affectifs) [Giuliani et Feldman, 1993 ; Bonaiuto et al., 1999; Hidalgo et Hernández, 2001; Lewicka, 2011], place dependence (aspects fonctionnels, usages) [Stokols et Shumaker, 1981], place identity (aspects identitaires) (Proshansky et al., 1983; Twigger-Ross et Uzzell, 1996; Bonaiuto et al., 2002). Un autre concept (place meaning), qui diffère de la notion d'attachement, est aussi utilisé pour décrire la signification symbolique de la relation au lieu (Brehm et al., 2013).

La distinction entre ces quatre concepts n'est pas toujours claire (Giuliani et Feldman, 1993; Hidalgo et Hernández, 2001; Stedman, 2002; Lewicka, 2011) en raison de la multiplicité des référents théoriques et des méthodes utilisées (Stedman, 2002; Hernandez et al., 2007 ; Lewicka, 2011). De plus, cette classification peut recouper certaines typologies utilisées en géographie ou s'en différencier (Seamon et Sowers, 2008; Moles, 1995). Cependant, géographes comme psychologues de l'environnement considèrent que, sous couvert du concept général de relation au lieu, il est important de distinguer plusieurs modalités pour mieux comprendre comment des individus ou des groupes appréhendent le risque. Il faut en effet entrer dans le détail de la dimension affective, identitaire, symbolique, ou dépendante pour que cette relation soit signifiante.

\section{Attachement au lieu (place attachment)}

L'attachement au lieu est un concept qui décrit la relation affective, les liens émotionnels avec un lieu particulier (Altman et Low, 1992). Dès lors, il correspond à la notion de topophilie utilisée en géographie. En psychologie environnementale, l'attachement au lieu fait l'objet de publications croissantes (Bonaiuto et al., 1999) avec deux revues majeures en la matière (Journal of Environmental Psychology et Environment and Behavior) [Lewicka, 2011].

Les origines de l'attachement au lieu viendraient, pour partie, des théories de la psychologie de l'attachement entre humains, en partant du développement de l'enfant et notamment des travaux sur l'attachement entre l'enfant et ceux qui en prennent soin. Cette théorie est centrée sur le désir de l'enfant de maintenir du lien et de la proximité avec l'objet de son attachement et sur l'impact de cette relation sur son bien-être (Bowlby, 1969; Hidalgo et Hernández, 2001). D’après Bowlby (1969), les êtres humains sont nés avec un système comportemental qui les motive à chercher la proximité de figures d'attachement pour se protéger de menaces. Parfois, le logement peut représenter une de ces figures de l'attachement, notamment pour les personnes âgées car il peut correspondre à la fois à une base de sécurité comme il peut constituer un lieu de la mémoire familiale.

Parmi les différentes formes d'attachement au lieu, l'enracinement (rootedness) [Lewicka, 2011] est une forme d'ancrage renforcée par la durée de résidence, la mémoire et la transmission intergénérationnelle (Kelly et Hosking, 2008). Une autre forme d'attachement est liée à la pratique du lieu, qu'il s'agisse de pratiques professionnelles, de loisirs ou d'interactions sociales.

\section{Signification du lieu (place meaning)}

La signification du lieu (place meaning) correspond à l'importance symbolique du lieu qui se réfère à l'expérience individuelle ou collective ou à la mémoire d'une expérience individuelle ou collective (Stedman, 2003; Brehm et al., 2013). Elle est le plus souvent estimée de façon qualitative (Jacobs et Buijs, 2011) en demandant aux enquêtés d'associer des mots à un lieu (Devine-Wright et Howes, 2010; Clarke et Mayer, 2017). Il est ainsi possible de distinguer quels sont les marqueurs symboliques associés à ce lieu et de repérer parmi eux les éléments géographiques, qu'ils soient biophysiques (la mer, l'île, la montagne, etc.) ou sociétaux (le patrimoine urbain, par exemple).

\section{Dépendance au lieu (place dependence)}

Lewicka (2011) définit comme "place dependence» la relation fonctionnelle qu'une personne peut entretenir avec son environnement. Cela comprend des éléments tels que l'accès aux soins de santé, le temps de trajet domicile-travail, ou encore l'accès aux installations de loisirs. Ce concept est lié aux usages que l'on fait du lieu. Il comprend ce que permet le lieu (notion d'affordance) 
du fait que certaines pratiques sont conditionnées par leur localisation. Il existe également une dimension utilitariste ou instrumentaliste de cette relation au lieu: un logement en bord de mer a ainsi potentiellement pour les propriétaires une forte valeur économique en cas de revente ou de location touristique et peut donc être perçu comme un bon placement financier.

\section{Identité par le lieu (place identity)}

L'identité par le lieu est un des éléments par lesquels un individu construit son identité au cours de sa vie (Fullilove, 1996). Elle participe de l'estime de soi, motive le comportement et contribue significativement au bien-être psychologique (Proshansky 1978; Twigger-Ross et Uzzell 1996; Manzo, 2003). Renvoyant à une dimension cognitive liée aux connaissances, aux croyances et aux liens sociaux, elle se différencie des dimensions affectives et symboliques. Twigger-Ross et Uzzell (1996) définissent quatre dimensions de l'identité par le lieu: la distinction, la continuité, l'estime de soi et l'efficacité personnelle. Le caractère distinctif correspond aux caractéristiques du lieu qui permettent à l'individu de se différencier des autres. La continuité se réfère aux relations passées avec le lieu qui sont maintenues dans le présent. L'estime de soi indique la capacité d'utiliser les caractéristiques du lieu pour améliorer l'image que l'on a de soi. L'efficacité personnelle est la capacité à répondre à des situations. Pour les auteurs, le concept d'identité peut renvoyer aux représentations sociales (Moscovici, 1976) en ce sens qu'elles donnent aussi au groupe sa spécificité et impliquent un ensemble de valeurs et de normes (Abric, 2001).

\section{Lien entre concernement et relation au lieu}

Peu d'articles font le lien entre la relation au lieu et le concernement (Brehm et al., 2013). La plupart des synthèses sur la relation au lieu et le concernement, notamment environnemental, constatent que de nombreuses contributions scientifiques ont cherché à expliquer le concernement environnemental à partir de variables sociodémographiques (âge, statut économique, durée de présence...) mais que les résultats obtenus sont contradictoires, incitant à approfondir l'analyse, et ce d'autant plus que des proximités théoriques existent entre ces deux concepts. En effet, la relation au lieu, comme le concernement, se réfèrent à la notion de sécurité ontologique. Ainsi, Tim Harries (2008) utilise cette notion pour expliquer pourquoi ne pas changer d'habitat en situation de risque peut être considéré comme une attitude rationnelle. La thèse associée est que les informations incompatibles avec la représentation du logement en tant que lieu de sécurité sont filtrées et que leur portée est minorée par les habitants (Michel-Guillou et al., 2016). Cette rationalité alternative à l'adaptation (où l'inaction est une forme de maintien du bien-être) a été observée par De Dominicis et al. (2015) à propos du risque d'inondation dans deux villes italiennes: lorsque l'attachement au lieu est fort, la relation entre perception $\mathrm{du}$ risque et action est amoindrie.

Brehm et al. (2013), ayant conduit une analyse sur les liens entre relation au lieu et concernement environnemental, constatent que peu de travaux s'intéressent au lien entre la relation au lieu et la dimension cognitive du concernement, et que la relation porte surtout sur les attitudes. Ils s'appuient sur les études suivantes. En Italie, Bonaiuto et al. (2002) comparent les attitudes des autochtones et allochtones et trouvent des relations différentes entre identité régionale, attachement au lieu et attitudes envers les parcs nationaux. Uzzell et al. (2002) ont analysé la relation entre cohésion sociale, identité par le lieu et attitudes pro-environnementales. Ils montrent que des communautés soudées ont de plus fortes identités médiées par le lieu (place identity) qui impliquent des attitudes pro-environnementales. En Norvège, l'étude des perceptions d'un projet hydroélectrique (Vorkinn et Riese, 2001) montre que l'attachement au lieu explique davantage la diversité des attitudes que les variables sociodémographiques. Enfin, Brehm et al. (2006) ont analysé les liens entre deux types d'attachement au lieu (social et environnemental) et les attitudes vis-à-vis de l'environnement: ils montrent une relation entre l'attachement social et des attitudes orientées vers les questions de santé, tandis que l'attachement environnemental est associé à des attitudes tournées vers la protection des ressources.

Par ailleurs, Armstrong et Stedman (2018) montrent que le concernement environnemental des résidents secondaires est construit par les relations sociales et psychologiques à propos du lieu. Selon Weiss et al. (2006), ce sont les personnes très attachées à leurs logements, dans lesquels elles ont vécu longtemps, qui sont les plus informées et impliquées dans la gestion de leur environnement.

Dans une étude qui distingue les formes de relations au lieu relatives à la perception des promeneurs sur la dégradation des sentiers dans les Appalaches, Kyle et al. (2004) observent que le concernement est lié à deux dimensions précises, la dépendance au lieu et l'identité par le lieu. Par ailleurs, Brehm et al. (2013) montrent la nécessité d'analyser la signification du lieu (place meaning) pour comprendre le renforcement des attitudes pro-environnementales.

Ces analyses des liens entre la relation au lieu et le concernement environnemental mettent en lumière que l'analyse ne peut se cantonner à la relation entre «relation au lieu» et «concernement» mais qu'il faut regarder plus précisément quelles sont les dimensions de 
Tab. 2. Questionnaires des différents projets.

\begin{tabular}{|c|c|c|c|c|c|c|c|}
\hline \multicolumn{8}{|c|}{ Méditerranée } \\
\hline $\begin{array}{l}\text { Zones } \\
\text { Enquêtées }\end{array}$ & Date & Risque & Population & Effectif & Passation & Durée & Nom \\
\hline $\begin{array}{l}\text { Languedoc- } \\
\text { Roussillon } \\
\text { (Palavas, Pérols, } \\
\text { Mauguio) } \\
\end{array}$ & 2009 & $\begin{array}{l}\text { Submersion } \\
\text { et érosion }\end{array}$ & $\begin{array}{l}\text { Principaux et } \\
\text { secondaires }\end{array}$ & 563 & $\begin{array}{c}\text { Face à } \\
\text { face } \\
\text { domicile }\end{array}$ & $45 \mathrm{mn}$ & Miseeva \\
\hline $\begin{array}{l}\text { Languedoc- } \\
\text { Roussillon } \\
\text { (Lattes, } \\
\text { Sommières) }\end{array}$ & 2018 & Inondation & $\begin{array}{l}\text { Principaux et } \\
\text { secondaires }\end{array}$ & 370 & $\begin{array}{c}\text { Face à } \\
\text { face } \\
\text { domicile } \\
\text { et dans la } \\
\text { rue }\end{array}$ & $40 \mathrm{mn}$ & MAGIC \\
\hline \multicolumn{8}{|c|}{ Atlantique/Manche } \\
\hline Lacanau & 2013 & $\begin{array}{l}\text { Érosion } \\
\text { plage }\end{array}$ & $\begin{array}{c}\text { Résidents } \\
\text { principaux } \\
\text { secondaires } \\
\text { Touristes et } \\
\text { excursionnistes }\end{array}$ & 507 & $\begin{array}{l}\text { Face à } \\
\text { face } \\
\text { (domicile } \\
\text { et rue) }\end{array}$ & $\begin{array}{c}20 \text { à } \\
30 \mathrm{mn}\end{array}$ & Lacanau \\
\hline $\begin{array}{l}5 \text { communes } \\
\text { Bretagne } \\
\text { (Guisseny, Sein, } \\
\text { L'île-Tudy, Le } \\
\text { Tour-du-Parc, } \\
\text { Penestin) }\end{array}$ & 2012 & $\begin{array}{l}\text { Submersion } \\
\text { et érosion }\end{array}$ & $\begin{array}{l}\text { Principaux et } \\
\text { secondaires }\end{array}$ & 894 & $\begin{array}{c}\text { Face à } \\
\text { face } \\
\text { domicile }\end{array}$ & $\begin{array}{c}30 \text { à } \\
45 \mathrm{mn}\end{array}$ & Cocorisco \\
\hline \multicolumn{8}{|l|}{ National } \\
\hline $\begin{array}{l}227 \text { communes } \\
\text { littorales de } \\
\text { France } \\
\text { métropolitaine }\end{array}$ & 2017 & Submersion & $\begin{array}{l}\text { Résidents } \\
\text { principaux }\end{array}$ & 782 & En ligne & $20 \mathrm{mn}$ & BURT \\
\hline
\end{tabular}

relations au lieu et les modalités du concernement qui peuvent être associées.

\section{Principes et modalité de comparaison des enquêtes et méthodologie d'évaluation des corrélations entre relation au lieu et concernement}

En lien avec les observations issues de la revue de littérature, et à partir d'un traitement comparatif de données obtenues lors de plusieurs enquêtes de terrain pour différents projets de recherche en zones côtières en France métropolitaine (Tab. 2), nous avons cherché à étudier les liens existants empiriquement entre la relation au lieu et le concernement d'individus face aux risques côtiers. Nous traitons donc ici des données secondaires, issues d'enquêtes effectuées dans des projets conçus et réalisés indépendamment. Bien que le projet MASALA regroupe une proportion importante des enquêtes conduites en France métropolitaine sur le risque côtier, nous ne pouvons prétendre que notre analyse sur plusieurs enquêtes contrôle la représentativité des enquêtés à l'échelle nationale. Nous identifions, au sein de chaque enquête quelles sont les relations significatives entre concernement et relation au lieu, puis nous regardons sur l'ensemble des enquêtes quels sont les types de relations qui ont été identifiés. Nous tirons de cet ensemble de relations les leçons qualitatives sur les interactions entre les deux concepts. Bien que les problématiques des enquêtes mobilisées n'aient pas toutes été centrées sur cette question du lien entre relation au lieu et concernement, un nombre suffisant de questions permettant de renseigner ces relations a pu être identifié et traité sur le plan statistique pour chaque enquête (Tab. 3 et 4 ). Nous détaillons ci-dessous la façon dont la relation au lieu d'une part, et le concernement visà-vis des risques côtiers d'autre part, a été inférée dans les questionnaires d'enquête et les données empiriques. Nous exposons ensuite les traitements et analyses effectués pour apprécier les relations entre ces deux composantes. 
Tab. 3. Questions portant sur les différentes dimensions de relation au lieu.

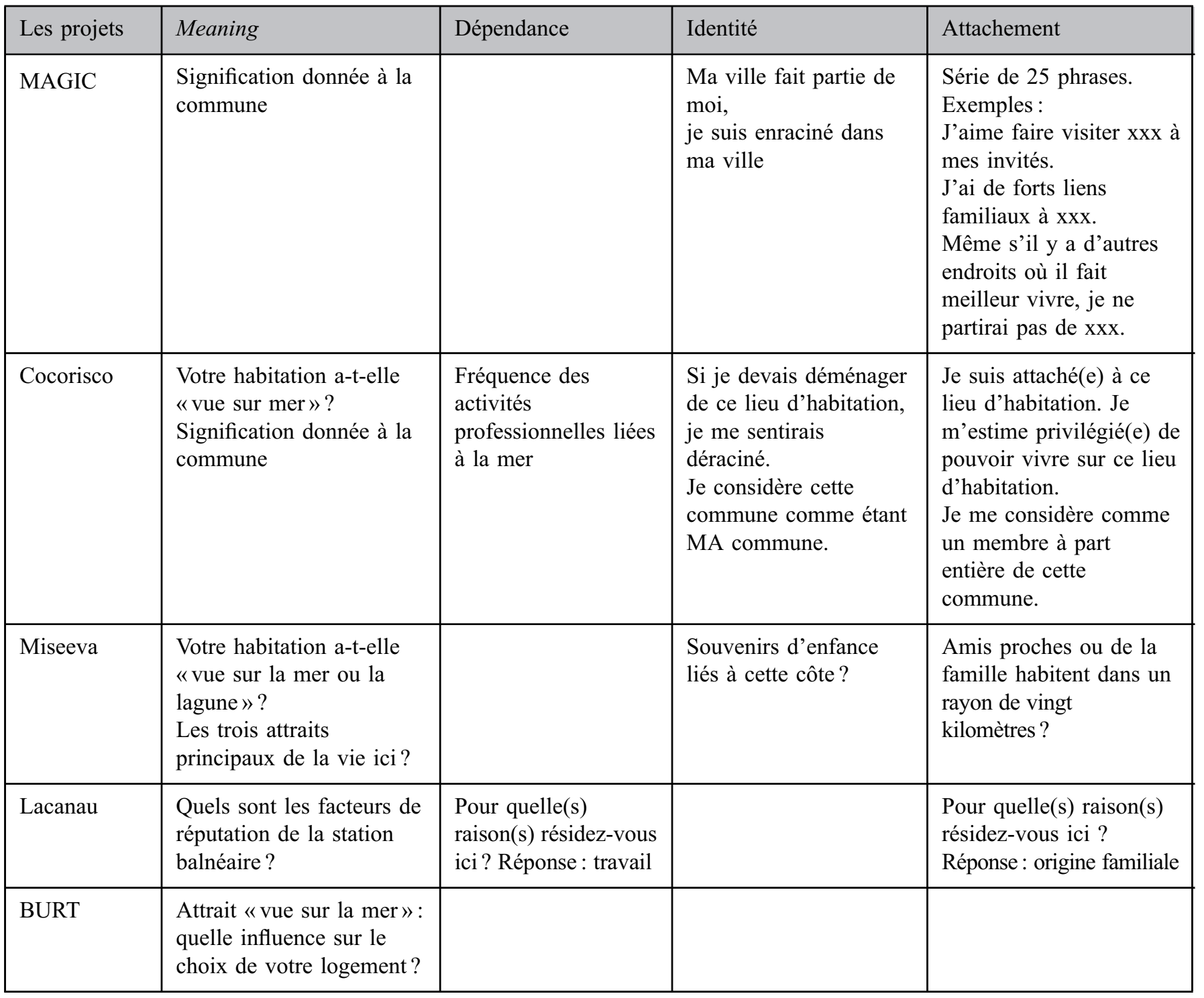

\section{La relation au lieu}

L'analyse a été menée en sélectionnant les questions rendant compte de la relation au lieu en les classant parmi les quatre dimensions que nous avons identifiées. Seules les questions pouvant être clairement liées à la relation au lieu ont été retenues, sachant que certaines questions pouvaient se référer à plusieurs dimensions de relations au lieu. Ce sont les auteurs de chaque enquête qui ont identifié quelles questions de leur questionnaire pouvaient être rattachées à une des quatre dimensions. Une personne de l'équipe a participé à toutes ces opérations pour assurer la cohérence générale des classements en dimensions. Le tableau 3 illustre quelques exemples de questions associées aux différentes dimensions de relation au lieu, présentes dans plusieurs questionnaires. Par exemple, ce sont des questions sur la vue sur mer et sur la valeur du cadre de vie, sur les préférences résidentielles ou plus généralement une association libre de mots liés à la commune qui rendent compte du place meaning. La dépendance au lieu (place dependance) est appréhendée essentiellement par des modalités de réponse à des questions rendant compte d'une relation fonctionnelle, par exemple une activité professionnelle liée à la mer. Pour l'identité par le lieu, plusieurs questions abordent directement cette notion (par exemple: «Je considère cette commune comme MA commune») tandis que dans d'autres cas ce sont certaines modalités de réponse aux questions qui font indirectement référence à l'identité (souvenirs d'enfance, 
Tab. 4. Questions portant sur le concernement.

\begin{tabular}{|c|c|c|}
\hline Projets & Concernement passif & Concernement actif \\
\hline MAGIC & $\begin{array}{l}\text { Conscience du risque }: \text { ma ville/quartier/ } \\
\text { propriété est à risque } \\
\text { Connaissance des inondations } \\
\text { Impacts de ces crues } \\
\text { (sont-elles positives ou négatives)? } \\
\text { Soutien aux actions suivantes: } \\
\text { au sujet de la gestion des inondations? }\end{array}$ & $\begin{array}{l}\text { Actions envisagées: } \\
\text { «Je peux faire quelque chose à propos de ...» } \\
\text { Connaissance des mesures pour réduire l'exposition } \\
\text { au risque inondation. } \\
\text { Liste des actions prises ou intention de prendre }\end{array}$ \\
\hline Cocorisco & $\begin{array}{l}\text { Je m'estime concerné(e) par les } \\
\text { décisions qui sont prises pour cette } \\
\text { commune } \\
\text { Conscience du risque: } \\
\text { Mots ou expressions sur «Risques sur } \\
\text { votre commune» «Érosion» et } \\
\text { d'« inondation marine». } \\
\text { Que pensez-vous du risque FUTUR de } \\
\text { montée du niveau marin? } \\
\text { Êtes-vous dans une zone dite «à } \\
\text { risque»? } \\
\text { Connaissances: } \\
\text { Risques côtiers ACTUELS (érosion/ } \\
\text { inondation marine) sur votre commune? } \\
\text { Éléments qui AUGMENTENT ou } \\
\text { DIMINUENT le risque d'érosion et/ou } \\
\text { d'inondation. }\end{array}$ & $\begin{array}{l}\text { Information et communication: } \\
\text { Quelles sources d'information(s) concernant les risques } \\
\text { côtiers sur votre commune? }\end{array}$ \\
\hline Miseeva & $\begin{array}{l}\text { Conscience du risque: } \\
\text { Que pensez-vous du risque de montée } \\
\text { du niveau de la mer pour la région? } \\
\text { Connaissances: } \\
\text { Comment imaginez-vous le lido et les } \\
\text { étangs à l'horizon } 2100 \text { ? } \\
\text { Risques futurs liés à la montée du } \\
\text { niveau de la mer dans les vingt ans à } \\
\text { venir? }\end{array}$ & $\begin{array}{l}\text { Actions envisagées: } \\
\text { Indemnisation/dédommagement: Avez-vous été } \\
\text { indemnisé(e)? OU Pensez-vous que vous seriez } \\
\text { indemnisé(e) en cas de dégâts? } \\
\text { Coût envisagé des systèmes de défense pour être } \\
\text { protégé face à la submersion marine. } \\
\text { Information et communication: } \\
\text { Avec qui avez-vous déjà parlé du risque d'inondation } \\
\text { marine? }\end{array}$ \\
\hline Lacanau & $\begin{array}{l}\text { Conscience des risques: } \\
\text { Les trois principaux risques } \\
\text { Connaissances: } \\
\text { Observation du littoral, changements, } \\
\text { quels effets? logement menacé par } \\
\text { l'érosion? Quelles gestions et } \\
\text { protections actuelles? Stratégie } \\
\text { régionale et gestion de la bande côtière? } \\
\text { Aspect administratif et juridique? }\end{array}$ & $\begin{array}{l}\text { Actions envisagées: } \\
\text { Que feriez-vous si plus de plage dans } X \text { années? } \\
\text { Conscience des actions/gestions envisageables } \\
\text { Information et communication: } \\
\text { Participation à des réunions publiques }\end{array}$ \\
\hline BURT & $\begin{array}{l}\text { Conscience du risque: } \\
\text { Que craignez-vous personnellement } \\
\text { d'une fréquence accrue des submersions } \\
\text { marines? } \\
\text { Êtes-vous sensible aux alertes rouges de } \\
\text { la météo? }\end{array}$ & $\begin{array}{l}\text { Actions envisagées: } \\
\text { Types d'actions/aménagements appropriés pour } \\
\text { s'adapter à cette montée du niveau de la mer? } \\
\text { Connaissance de la relocalisation : Êtes-vous pour la } \\
\text { relocalisation des habitations les plus menacées par le } \\
\text { risque submersion? }\end{array}$ \\
\hline
\end{tabular}


Tab. 4. (suite).

\begin{tabular}{|c|c|c|}
\hline Projets & Concernement passif & Concernement actif \\
\hline & $\begin{array}{l}\text { Pensez-vous que votre habitation } \\
\text { pourrait être concernée par une } \\
\text { relocalisation? } \\
\text { Comment qualifier la relocalisation : une } \\
\text { utopie; une opportunité; un espoir; un } \\
\text { préjudice; une menace, inévitable }\end{array}$ & $\begin{array}{l}\text { Quels sont les critères qui vous semblent les plus } \\
\text { importants pour que ces politiques de recul soient } \\
\text { acceptées par la population? } \\
\text { Financement/coût imposition: } \\
\text { Pour la relocalisation et l'indemnisation des biens } \\
\text { privés (habitat et activités)? } \\
\text { Êtes-vous prêt à payer plus d'impôts pour financer la } \\
\text { politique de relocalisation? } \\
\text { Information et communication: } \\
\text { Recherche active de l'information? }\end{array}$ \\
\hline
\end{tabular}

histoire de la commune). Enfin, de nombreuses variables portent directement sur l'attachement (exemple : « Je suis attaché » à ce lieu), mais il peut aussi s'agir d'indicateurs indirects de l'attachement comme le temps de résidence.

\section{Le concernement}

De la même façon, le concernement a été appréhendé suivant différentes modalités construites de manière inductive à partir des enquêtes et des résultats de terrain disponibles. Schématiquement, quatre modalités se déclinent en croisant différents degrés de concernement: la présence ou l'absence d'un concernement (indiqué ici «non-concernement»), et son caractère plus ou moins «passif» ou «actif» (Tab. 1). Le nonconcernement peut alors soit relever d'une forme passive (ignorance du risque) ou active (par la mise à distance d'une situation problématisée à risque). En outre, si le concernement "passif» peut s'observer en relevant une connaissance ou une expérience sensible du risque, le concernement "actif» donne lieu à des formes de mobilisation, de veille et de recherches d'information, ou encore de discussions avec autrui. C'est dans ce type de situation que peuvent advenir des processus de publicisation, de construction d'un problème public par l'engagement d'acteurs sociaux (Cefaï, 1996). C'est à ce titre que ces modalités de concernement interagissent en faisant notamment transparaître le rôle des relations sociales : de « dispersé, privé et silencieux», ce concernement peut devenir «collectif, public et organisé» (Brunet, 2008).

Afin de mettre à l'épreuve cette grille de lecture, nous avons, de même que pour la relation au lieu, identifié des questions au sein des enquêtes disponibles permettant de qualifier et de caractériser ces degrés de concernement vis-à-vis des risques littoraux et fluviaux (Tab. 4). Pour le concernement passif, deux modalités de réponses ont été retenues :
- La première, relative à l'appréhension des phénomènes et à la sensibilité aux risques (la conscience), à différentes échelles, indique ou peut indiquer un trouble vis-à-vis de la «sécurité ontologique» (par exemple: pour moi, ma ville/mon quartier/mon logement est à risque);

- La seconde est relative à la connaissance de ces phénomènes et de leurs conséquences (exemple: quels sont les éléments qui augmentent ou diminuent le risque d'érosion et/ou de submersion/inondation?).

Pour le concernement actif, deux autres modalités de réponse ont été mobilisées selon qu'il s'agit:

- des dynamiques personnelles (et plus ou moins actives) d'information et de communication (exemple : Avez-vous participé à des réunions publiques sur ce sujet ?);

- des actions collectives envisagées ou effectivement réalisées (exemple : Suite à la tempête Xynthia, avezvous participé à une ou plusieurs actions?).

\section{Tester les liens entre relation au lieu et concernement}

L'étape suivante fut d'identifier, pour chaque enquête, quelles étaient les relations statistiquement significatives entre les différentes dimensions de la relation au lieu et les différentes modalités du concernement. L'analyse a été menée à partir de tests du Khi 2 pour tester l'existence d'une corrélation entre les deux types de variables à l'échelle de chaque enquête. Les résultats ont été partagés au sein du groupe de chercheurs et une personne de référence a regroupé les interprétations et homogénéisé leur présentation. Les relations significatives ont été dénombrées en distinguant si elles s'effectuaient de façon positive ou négative. Le tableau 5 présente ainsi le résultat du croisement des différentes enquêtes. 
Tab. 5. Nombre de relations significatives dans les enquêtes (en gris les relations positives, en noir les relations négatives).

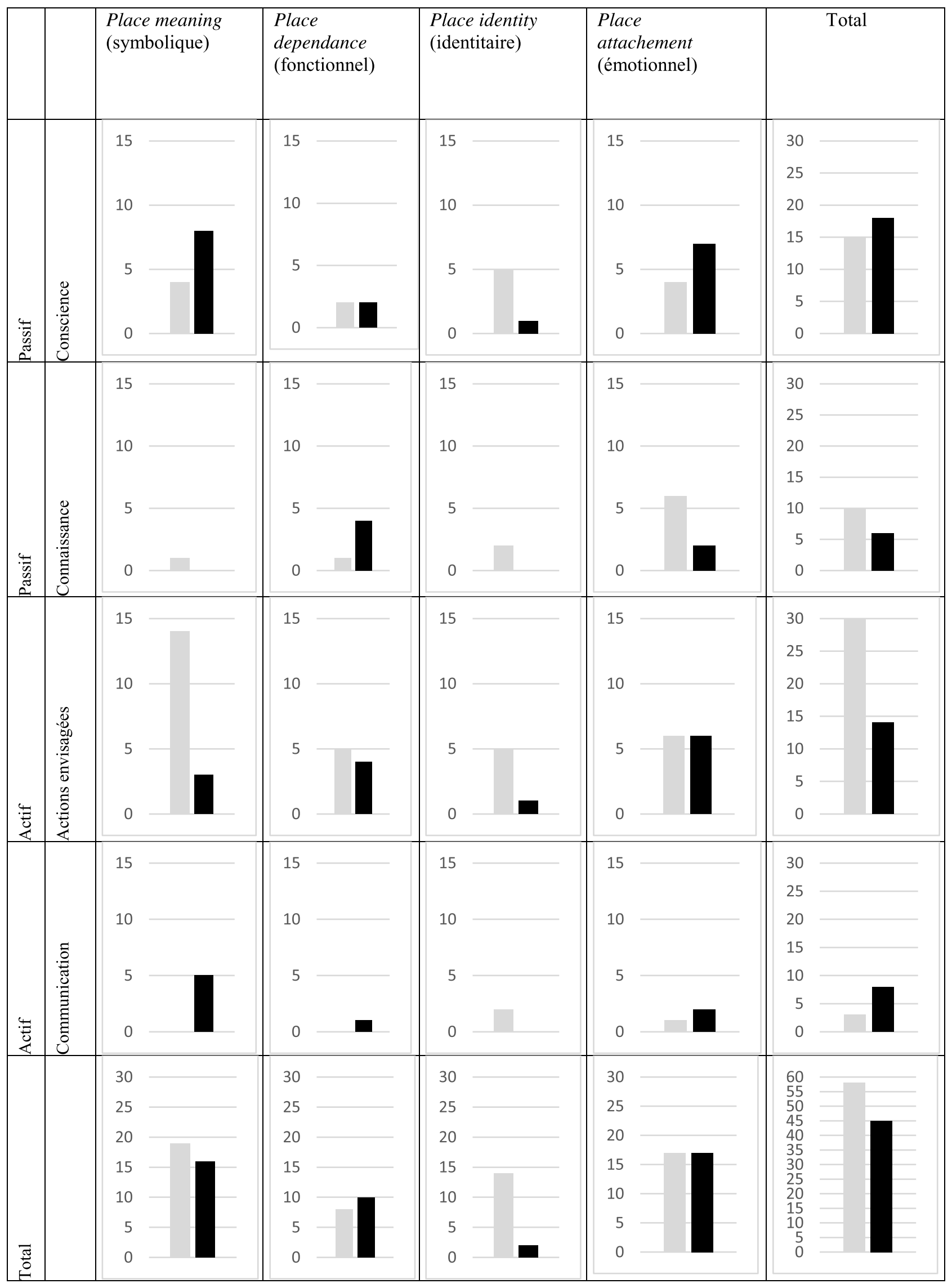


Tab. 6. Exemples de relations négatives entre dimensions symboliques et émotionnelles de la relation au lieu et sensibilité aux risques côtiers (source: tests de Khi 2 menés sur les données d'enquête).

Ceux qui habitent près de la mer (quartier mer) sont plus souvent sceptiques quant au risque de submersion.

Ceux qui sont dans leur logement depuis plus de 20 ans sont plus souvent sceptiques sur le risque de submersion.

Ceux qui sont là depuis plus de 20 ans ont plus souvent le sentiment de n'être pas exposés.

Plus le score d'attachement (synthétisant de nombreuses questions sur cette modalité) est fort, moins forte est l'appréciation du risque.

Si les biens sont acquis par héritage, les enquêtés sont moins inquiets pour les dégâts sur leur commune.

Si la motivation d'habiter est «mer et nature», les enquetés ne sont pas d'accord avec l'affirmation de l'existence d'un risque actuel et futur.

Si la motivation d'habiter est «mer et nature», les enquêtés pensent davantage que «rien ne prouve que cela arrivera ».

Si la motivation d'habiter est «tranquillité », les enquêtés ne sont pas d'accord avec l'affirmation de l'existence d'un risque actuel et futur.

Si la représentation de la commune est «commune belle, balnéaire, maritime», les enquêtés ne sont pas d'accord avec l'affirmation de l'existence d'un risque actuel et futur.

Si la représentation de la commune est «commune belle, balnéaire, maritime», les enquêtés pensent davantage que «rien ne prouve que cela arrivera».

Si la représentation de la commune est «commune belle, balnéaire, maritime», les enquêtés sont moins inquiets pour les dégâts sur leur commune.

Ceux qui citent «tranquillité» dans «attraits principaux de la vie ici», pensent moins que le lido pourrait se rompre à cause de la remontée du niveau de la mer.

\section{Interprétation des résultats et discussion}

\section{Des relations plurielles entre relation au lieu et concernement}

Conformément aux observations issues de la revue de littérature, les résultats témoignent de relations non univoques, où des types de relations au lieu peuvent favoriser la prise en compte du risque ou bien au contraire contribuer à sa mise à distance. En effet, l'inventaire des relations positives (58) [une plus forte relation au lieu est corrélée à un plus fort concernement] et négatives (45) [une plus forte relation au lieu est corrélée à un concernement moins fort] confirme ce caractère en apparence contradictoire et oblige à étudier plus précisément les dimensions de relations au lieu et les modalités de concernement. Il est intéressant ici de se concentrer sur les deux relations à la fois les plus étudiées dans la littérature et pour lesquelles le nombre d'observations est le plus important: le place meaning (relation d'ordre symbolique) et l'attachement au lieu (relation émotionnelle). Ces deux dimensions sont analysées au regard du concernement passif tout d'abord, puis de modalités plus actives du concernement.

\section{Les relations au lieu d'ordre symbolique et émotionnel seraient associées à une moindre sensibilité aux risques}

Précisons à ce stade que les liens testés ici ne sont pas des relations de causalité mais des tests de corrélations entre deux variables ou modalités de réponse issues des questionnaires d'enquête. Les corrélations observées entre modalités symboliques (place meaning) et émotionnelles (place attachment) de la relation au lieu, d'une part, et du concernement passif, d'autre part, montrent ainsi plutôt un lien négatif, dans la mesure où 15 corrélations sont négatives et 8 sont positives (Tab. 5). Des exemples empiriques de corrélations négatives sont donnés dans le tableau 6 . Pour illustration: «Plus le score d'attachement (synthétisant de nombreuses questions sur cette modalité) est fort, moins forte est l'appréciation du risque », «Si la représentation de la commune est "commune belle, balnéaire, maritime", les enquêtés sont moins inquiets pour les dégâts sur leur commune». 
Tab. 7. Exemples de relations positives entre dimension symbolique de la relation au lieu et concernement actif.

Ceux qui habitent près de la mer (quartier mer) se sentent concernés par la relocalisation.

Ceux qui ont pour motivation mer et nature sont davantage contre la relocalisation.

Ceux qui ont pour motivation la convivialité veulent davantage consolider les ouvrages.

Ceux qui ont pour motivation la convivialité acceptent davantage de payer pour les ouvrages.

Ceux pour qui la commune est belle, balnéaire, maritime veulent davantage consolider les ouvrages.

Ceux qui vont à la plage ont plus tendance à chercher de l'information.

Ceux qui pratiquent le surf ont plus tendance à être prêts à contribuer financièrement.

Ceux qui ont cité les espaces naturels comme facteur de la réputation de Lacanau ont plus tendance à penser que les associations, les habitants et professionnels de Lacanau, et les personnes dont les biens sont menacés sont légitimes...

Si l'on se réfère à la littérature, on retrouve ici la notion de sécurité ontologique (Giddens, 1984 ; Barbier et Nadaï, 2015) au sens où le lieu dans lequel on vit doit être perçu comme un lieu de sécurité et les informations qui tendent à montrer le contraire sont alors minorées. Une relation au lieu d'ordre symbolique et/ou émotionnel contribuerait ainsi davantage à la mise à distance des phénomènes qui viennent troubler cet espace de sécurité et à la diminution du concernement. "Applying the idea of ontological security to home, home presents a representational barrier through which information must pass and the power and resonance of incongruent information that challenges the security of home is therefore reduced. » (Quinn et al., 2018).

\section{La relation d'ordre symbolique favoriserait le concernement actif}

Concernant les effets du rapport symbolique au lieu (place meaning), on observe 17 relations dont 14 corrélées avec un concernement actif (Tab. 5), quelques exemples étant proposés dans le tableau 7. En voici un: «Ceux pour qui la commune est belle, balnéaire, maritime, veulent davantage consolider les ouvrages ». Cette relation génère l'effectif le plus important de relations significatives (positives ou négatives) dans les enquêtes analysées. On peut la rapprocher de certains travaux de la littérature montrant que la relation au lieu influence parfois des mobilisations ou des actions vis-à-vis d'un risque donné, soit en les minorant, soit en les favorisant comme dans les résultats présents (Bonaiuto et al., 2016). Il apparaît cependant que, lorsque la relation au lieu favorise un concernement actif (Tab. 6), c'est le plus souvent pour préserver une forme de statu quo, soit en consolidant des ouvrages de défense contre l'érosion et/ou la submersion, soit en s'opposant à la relocalisation des biens et des activités. Ces types d'actions confirment les constats réalisés par Clarke et al. (2018) qui soulignent que "Transformation is likely to be resisted by individuals if it negatively disrupts place». La plupart des recherches montrent en effet des situations où la relation au lieu constitue plutôt un frein au changement (Michel-Guillou et al., 2016). Par ailleurs, d'autres travaux mettent en évidence que certaines représentations symboliques peuvent favoriser l'action en faveur du changement, à condition que ce changement soit en harmonie ou en accord avec ces représentations. Quinn et al. (2019) montrent, à propos des risques d'inondation et de submersion, que selon leur représentation symbolique, les personnes réagissent différemment aux divers risques. Ils acceptent par conséquent plus ou moins que différents types d'actions soient entrepris pour se protéger de l'inondation ou faire face à ses effets.

Ainsi, les résultats de la présente recherche mettent en avant, dans la continuité de certains travaux et pour le cas des littoraux français, que la relation au lieu peut en même temps contribuer à la mise à distance des risques encourus et concourir au concernement actif à propos de ces mêmes risques. L'explication de ce paradoxe apparent renvoie à la fois à une analyse plus fine des dimensions de l'attachement, à l'histoire des personnes dans ce lieu, aux activités pratiquées (exemple du surf) et à la différence entre perception individuelle et perception collective. Une personne peut en effet minorer le risque pour elle-même et s'engager pour le risque que subirait le collectif. À partir de l'enquête menée à Lacanau, Rocle et al. (2016) ont ainsi relevé un « concernement local» et collectif au regard de la situation-problème engendrée par l'érosion marine sur cette commune.

\section{Discussion et implications pour l'aide à la décision}

Ces apparentes divergences ont des conséquences sur l'action menée dans les territoires et sur les formes d'aménagement et de gestion des risques d'érosion, d'inondation et de submersion. Comme l'affirment 
Clarke et al. (2018): "To reduce or overcome disruptions, place-based identities and meanings should be incorporated into policy and planning processes [...]. Understanding emotional place-related values early in the adaptation process may contextualise attributes of place by detailing what aspects cause concern where disruptions are proposed $\gg$. Ainsi, à la suite de Masterson et al. (2017), deux perspectives issues de cette analyse des relations au lieu peuvent permettre de tirer des leçons pour l'aide à la décision. Il s'agit, d'une part de la question de la transformation des relations au lieu et, d'autre part, de la prise en compte de la multiplicité des relations au lieu au moyen de dispositifs participatifs.

Lorsque des individus reconnaissent le besoin de changement mais que les valeurs associées aux lieux sont en contradiction avec ces changements, une voie possible est d'agir pour favoriser une transformation cognitive afin de dépasser la dissonance existante entre les désirs paradoxaux de stabilité et les désirs de changement. De nombreux auteurs s'intéressent à ce titre à l'évolution des relations au lieu et en particulier à l'évolution des relations symboliques. Ainsi, Séverine Durand (2014) montre comment la relation des habitants de la ville de Lattes au fleuve côtier Lez a changé au cours des 20 dernières années, sous l'effet (i) de la construction d'infrastructures pour endiguer et contrôler le fleuve, favorisant une moindre appréhension des phénomènes d'inondation; (ii) d'une communication institutionnelle mettant à distance le fleuve; et (iii) du changement de composition démographique de la ville, avec l'arrivée de nouveaux habitants favorisée justement par ce contrôle du fleuve. Des processus de communication visant à sensibiliser et à modifier les perceptions ou représentations d'un risque ne sont alors pas suffisants dans la mesure où l'attachement au lieu est aussi une construction sociale issue des rapports entre différents acteurs (résidents, élus, aménageurs, etc..). Au-delà d'une « construction sociale du risque», c'est donc davantage un «construit territorial» du risque qu'il s'agit d'appréhender, car les dimensions spatiales et identitaires (mais également de dépendance au lieu quand il s'agit d'économie territoriale) jouent fortement (Vinet, 2007).

À ce titre, Clément Mabi (2014) insiste, après avoir étudié les processus de concernement des publics pour les sujets discutés au sein de la Commission nationale du débat public, sur la formation d'une «relation de sens» entre une situation (ou un objet) et une personne, relation qui ne va pas de soi et qui résulte d'une «construction sociale issue des interactions entre les acteurs, leur environnement et l'objet du débat» (Mabi, 2014). Le concernement s'entend pour lui comme un «processus dynamique qui fait le lien entre l'expérience des individus et une situation problématique [...] dans l'environnement des individus. [II] ne se construit pas uniquement dans des dimensions sociales ou psychologiques, mais dans la manière dont la thématique est mise en débat, via des dispositifs de médiation, configure en partie l'expérience des publics » (Mabi, 2014). Les dispositifs d'information, de médiation (forum public à Lacanau [Rocle et al., 2016], festival «Si la mer monte» sur l'Île-Tudy, etc.) font partie de ces éléments de construction territoriale des risques côtiers et jouent aussi fortement sur l'évolution des relations au lieu.

En faisant du lien entre de multiples acteurs qui ont des relations au lieu différentes, ces dispositifs jouent ainsi sur la participation aux processus collectifs. Comme Clarke et al. (2018) le remarquent, ce sont surtout ceux qui ont une forte relation au lieu qui s'engagent plus fortement dans ce type de dispositifs participatifs. Comme exposé supra, ces engagements peuvent porter sur des mesures d'adaptation souhaitées (par exemple de relocalisation) par des gestionnaires et élus locaux, mais peuvent aussi chercher à renforcer un statu quo ou à défendre des positions allant à l'encontre des pouvoirs publics (opposition à un plan de prévention des risques, par exemple). La participation d'une diversité de personnes ayant différentes relations au lieu et différents degrés de concernement peut permettre l'expression de cette diversité et donner ainsi une visibilité aux individus ou aux groupes sociaux qui, de par un concernement plus passif ou faute de moyens d'accès à l'espace public, ne sont pas en mesure de faire entendre leurs positions ou leurs valeurs dans les débats publics consacrés à la gestion des risques côtiers. Ainsi la connaissance des valeurs (ici la relation au lieu) et leurs liens avec les formes de concernement et d'engagement des populations permet-elle de conduire de tels processus collectifs dans la perspective d'une réduction des inégalités face aux risques côtiers.

\section{Conclusion}

Ce travail de croisement et de synthèse de différentes recherches sur la relation au lieu et les risques en zones côtières et estuariennes a permis de proposer, en mobilisant la notion de concernement, une lecture plus fine des multiples relations au lieu et de leurs effets sur la représentation des risques d'érosion, de submersion ou d'inondation. Pour cela, à partir d'une revue de la littérature et de la mise en commun de plusieurs enquêtes réalisées sur ces questions en France métropolitaine, nous avons caractérisé les liens observés entre types de relation au lieu et types de concernement. La mobilisation de ce large éventail de travaux couvrant plusieurs façades maritimes permet d'identifier des types de situations qui confirment les relations complexes, voire ambivalentes au premier regard, entre relation au lieu et concernement. Ainsi, nous avons montré qu'une relation au lieu analysée au travers des dimensions symboliques 
(place meaning) et émotionnelles (place attachment) pouvait conduire à une faible sensibilité vis-à-vis d'un risque, tout en favorisant différents types d'actions (se mobiliser pour que rien ne change, se tenir informé et participer aux débats locaux, se mobiliser pour faire advenir une option d'adaptation, etc.) cohérentes avec cette mise à distance du risque. Au-delà des précautions relatives au sens à donner à ces corrélations statistiques, plusieurs auteurs mettent l'accent sur l'intérêt de ces analyses pour l'aide à la décision, à l'image de Cheng et Daniels (2003) : «Incorporating sense of place in natural resources and land use management is an important component of making acceptable decisions; especially at the local level ». Nos analyses portant sur les liens tissés entre les habitants et avec leur lieu de vie permettent de mieux comprendre leurs attitudes et leurs actions et, par conséquent, les processus de construction territoriale des risques en zones côtières. Engager dans ces processus des personnes ayant différentes relations au lieu et différents types de concernement peut alors favoriser l'expression de cette diversité sur les actions à entreprendre, mais peut également être une voie de réduction des inégalités environnementales afin de ne pas privilégier les individus et groupes sociaux démontrant les plus forts degrés de concernement et d'engagement.

Enfin, soulignons que cette réflexion constitue une expérience de travail interdisciplinaire via des regards croisés sur l'analyse de diverses études de terrain qui, pour la plupart, n'abordaient que ponctuellement ces problématiques. La mise en commun de la bibliographie et des différentes enquêtes entre chercheurs et entre disciplines a permis d'identifier des voies pour contribuer collectivement à la connaissance sur la relation au lieu et le concernement, en caractérisant de façon interdisciplinaire les interactions entre ces notions. Le cadre que nous proposons, qui distingue quatre dimensions de la relation au lieu et deux modalités de concernement, offre une voie pour expliquer des relations qui paraissent paradoxales au premier abord. Il pourrait maintenant être utilisé pour de futures enquêtes qui se doteraient d'un protocole et d'une stratégie d'échantillonnage permettant de produire des leçons statistiquement étayées. Ce cadre pourra aussi servir de référence pour des études qualitatives sur les relations entre des attachements, des émotions et des affects et le concernement environnemental.

\section{Références}

Abric J.-C., 2001. L'approche structurale des représentations sociales : développements récents, Psychologie et société, 2, 4, 81-104.

Altman I., Low S.M. (Eds), 1992. Place attachment, New York, Plenum Press.

Armstrong A., Stedman R.C., 2018. Understanding local environmental concern: the importance of place, Rural Sociology, 84, 1, 93-122, https://doi.org/10.1111/ruso.12215.
Bachelard G., 1957. La poétique de l'espace, Paris, Presses universitaires de France.

Barbier R., Nadaï A., 2015. Acceptabilité sociale: partager l'embarras, VertigO La revue électronique en sciences de l'environnement, 15, 3, https://doi.org/10.4000/vertigo.16686.

Bernardo F., 2013. Impact of place attachment on risk perception: exploring the multidimensionality of risk and its magnitude, Estudios de Psicología, 34, 3, 323-329, https:// doi.org/10.1174/021093913808349253.

Berque A., 2000. Écoumène. Introduction à l'étude des milieux humains, Paris, Belin.

Billig M., 2006. Is my home my castle? Place attachment, risk perception, and religious faith, Environment and Behavior, 38, 2, 248-265, https://doi.org/10.1177/0013916505277608.

Bonaiuto M.A., Aiello M., Perugini M., Bonnes M., Ercolani A.P., 1999. Multidimensional perception of residential environmental quality and neighbourhood attachment in the urban environment, Journal of Environmental Psychology, 19, 4, 331-352, https://doi.org/10.1006/ jevp.1999.0138.

Bonaiuto M., Carrus G., Martorella H., Bonnes M., 2002. Local identity processes and environmental attitudes in land use changes: the case of natural protected areas, Journal of Economic Psychology, 23, 5, 631-653, https://doi.org/ 10.1016/S0167-4870(02)00121-6.

Bonaiuto M., Alves A., De Dominicis S., Petruccelli I., 2016. Place attachment and natural hazard risk: research review and agenda, Journal of Environmental Psychology, 48, 3353, https://doi.org/10.1016/j.jenvp.2016.07.007.

Bowlby J., 1969. Attachment and loss. Vol. 1: Attachment, New York, Basic Books.

Brehm J.M., Eisenhauer B.W., Krannich R.S., 2006. Community attachments as predictors of local environmental concern: the case for multiple dimensions of attachment, American Behavioral Scientist, 50, 2, 142-165, https://doi. org/10.1177/0002764206290630.

Brehm J.M., Eisenhauer B.W., Stedman R.C., 2013. Environmental concern: examining the role of place meaning and place attachment, Society \& Natural Resources, 26, 5, 522-538, https://doi.org/10.1080/08941920.2012.715726.

Brunet P., 2008. De l'usage raisonné de la notion de « concernement»: mobilisations locales à propos de l'industrie nucléaire, Natures Sciences Sociétés, 16, 4, 317-325, https://doi.org/10.1051/nss/2008062.

Burley D., Jenkins P., Laska S., Davis T., 2007. Place attachment and environmental change in coastal Louisiana, Organization \& Environment, 20, 3, 347-366, https://doi. org/10.1177/1086026607305739.

Canguilhem G., 1992. La connaissance de la vie, Paris, Vrin.

Cefaï D., 1996. La construction des problèmes publics. Définitions de situations dans des arènes publiques, Réseaux, 75, 43-66, www.persee.fr/doc/reso_0751-7971_1996_ num_14_75 3684 .

Chateauraynaud F., 2007. La contrainte argumentative. Les formes de l'argumentation entre cadres délibératifs et puissances d'expression politiques, Revue européenne des sciences sociales, XLV-136, 129-148, https://doi.org/ 10.4000/ress.93. 
Cheng A.S., Daniels S.E., 2003. Examining the interaction between geographic scale and ways of knowing in ecosystem management: a case study of place-based collaborative planning, Forest Science, 49, 6, 841-854, https://academic.oup.com/forestscience/article/49/6/841/ 4617495? $\operatorname{login}=$ true.

Clarke D., Murphy C., Lorenzoni I., 2018. Place attachment, disruption and transformative adaptation, Journal of Environmental Psychology, 55, 81-89, https://doi.org/ 10.1016/j.jenvp.2017.12.006.

Clarke H.E., Mayer B., 2017. Community recovery following the Deepwater Horizon oil spill: toward a theory of cultural resilience, Society \& Natural Resources, 30, 2, 129-144, https://doi.org/10.1080/08941920.2016.1185556.

Dake K., 1991. Orienting dispositions in the perception of risk: an analysis of contemporary worldviews and cultural biases, Journal of Cross-Cultural Psychology, 22, 1, 61-82, https://doi.org/10.1177/0022022191221006.

Dardel E., 1952. L'homme et la terre. Nature de la réalité géographique, Paris, Presses universitaires de France.

De Dominicis S., Fornara F., Ganucci Cancellieri U., TwiggerRoss C., Bonaiuto M., 2015. We are at risk, and so what? Place attachment, environmental risk perceptions and preventive coping behaviours, Journal of Environmental Psychology, 43, 66-78, https://doi.org//10.1016/j. jenvp.2015.05.010.

Debarbieux B., 2012. Tourism, imaginaries and identities: reversing the point of view, Via. Tourism Review, https:// doi.org/10.4000/viatourism.1197.

Devine-Wright P., Howes Y., 2010. Disruption to place attachment and the protection of restorative environments: a wind energy case study, Journal of Environmental Psychology, 30, 3, 271-280, https://doi.org/10.1016/j. jenvp.2010.01.008.

Di Méo G., 2004. Composantes spatiales, formes et processus géographiques des identités, Annales de géographie, 339362, www.persee.fr/doc/geo_0003-4010_2004 num $113 \quad 63821628$.

Dietz T., Stern P.C., Guagnano G.A., 1998. Social structural and social psychological bases of environmental concern, Environment and Behavior, 30, 4, 450-471, https://doi.org/ 10.1177/001391659803000402.

Duclos D., 1987. La construction sociale du risque : le cas des ouvriers de la chimie face aux dangers industriels, Revue française de sociologie, 28, 1, www.persee.fr/doc/ rfsoc 0035-2969 1987 num 2812366.

Dunlap R.E., Van Liere K.D., 1978. The "new environmental paradigm", The Journal of Environmental Education, 9, 4, 10-19, https://doi.org/10.1080/00958964.1978.10801875.

Durand S., 2014. «Vivre avec la possibilité d'une inondation »? Ethnographie de l'habiter en milieu exposé... et prisé. Thèse de doctorat en sociologie, Marseille, Aix-Marseille Université.

Faburel G., Tribout S., 2011. Les quartiers durables sont-ils durables? De la technique écologique aux modes de vie, Cosmopolitiques, 19, 20, https://archive.boullier.bzh/cos mopolitiques_com/cosmopolitiques_com_archive boul lier_bzh_Quartiers\%20durables\%20et\%20 modes $\% 20 \mathrm{de} \%$
20vie\%20GF\%20et\%20ST\%20Mars\%202010\%20final. pdf.

Fransson N., Gärling T., 1999. Environmental concern: conceptual definitions, measurement methods, and research findings, Journal of Environmental Psychology, 19, 4, 369382, https://doi.org/10.1006/jevp.1999.0141.

Fullilove M.T., 1996. Psychiatric implications of displacement: contributions from the psychology of place, The American Journal of Psychiatry, 153, 12, 1516-1523, https://doi.org/10.1176/ajp.153.12.1516.

Giddens A., 1984. The constitution of society: outline of the theory of structuration, Berkeley, University of California Press.

Giuliani M.V., Feldman R., 1993. Place attachment in a developmental and cultural context, Journal of Environmental Psychology, 13, 3, 267-274.

Gold J.R., 1980. An introduction to behavioural geography, London/New York, Oxford University Press.

Hacking I., 2008. Entre science et réalité. La construction sociale de quoi ?, Paris, La Découverte.

Harries T., 2008. Feeling secure or being secure? Why it can seem better not to protect yourself against a natural hazard, Health, Risk \& Society, 10, 5, 479-490, https://doi.org/ 10.1080/13698570802381162.

Hay R., 1998. Sense of place in developmental context, Journal of Environmental Psychology, 18, 1, 5-29, https:// doi.org/10.1006/jevp.1997.0060.

Heidegger M., 1958. Bâtir, habiter, penser. Essais et conférences, Paris, Gallimard, 170-193.

Hellequin A.-P., Flanquart H., Meur-Ferec C., Rulleau B., 2013. Perceptions du risque de submersion marine par la population du littoral languedocien: contribution à l'analyse de la vulnérabilité côtière, Natures Sciences Sociétés, 21, 4, 385-399, https://doi.org/10.1051/nss/2014002.

Hernandez B.C., Hidalgo M.C., Salazar-Laplace M.E., Hess S., 2007. Place attachment and place identity in natives and non-natives, Journal of Environmental Psychology, 27, 4, 310-319.

Hidalgo M.C., Hernández B., 2001. Place attachment: conceptual and empirical questions, Journal of Environmental Psychology, 21, 3, 273-281, https://doi.org/10.1006/ jevp.2001.0221.

Jacobs M.H., Buijs A.E., 2011. Understanding stakeholders' attitudes toward water management interventions: role of place meanings, Water Resources Research, 47, 1, https:// doi.org/10.1029/2009WR008366.

Jørgensen B.S., Stedman R.C., 2001. Sense of place as an attitude: Lakeshore owners attitudes toward their properties, Journal of Environmental Psychology, 21, 3, 233-248, https://doi.org/10.1006/jevp.2001.0226.

Jørgensen B.S., Stedman R.C., 2006. A comparative analysis of predictors of sense of place dimensions: attachment to, dependence on, and identification with lakeshore properties, Journal of Environmental Management, 79, 316-327, https://doi.org//10.1016/j.jenvman.2005.08.003.

Kelly G., Hosking K., 2008. Nonpermanent residents, place attachment, and "sea change" communities, Environment and Behavior, 40, 4, 575-594, https://doi.org/10.1177/ 0013916507302246 . 
Kyle G., Graefe A., Manning R., Bacon J., 2004. Effects of place attachment on users' perceptions of social and environmental conditions in a natural setting, Journal of Environmental Psychology, 24, 2, 213-225, https://doi.org/ 10.1016/j.jenvp.2003.12.006.

Lefebvre H., 1974. La production de l'espace, Paris, Anthropos.

Lévy J., Lussault M., 2003. Lieu, in Lévy J., Lussault M. (Eds), Dictionnaire de la géographie et de l'espace des sociétés, Paris, Belin, 555-565.

Lewicka M., 2011. Place attachment: how far have we come in the last 40 years?, Journal of Environmental Psychology, 31, 3, 207-230, https://doi.org/10.1016/j.jenvp.2010.10.001.

Mabi C., 2014. Le débat CNDP et ses publics à l'épreuve du numérique: entre espoirs d'inclusion et contournement de la critique sociale. Thèse de doctorat en sciences de l'information et de la communication, Compiègne, Université de technologie de Compiègne.

Manzo L.C., 2003. Beyond house and haven: toward a revisioning of emotional relationships with places, Journal of Environmental Psychology, 23, 1, 47-61, https://doi.org/ 10.1016/S0272-4944(02)00074-9.

Masten A.S., Cicchetti D., 2016. Resilience in development: progress and transformation, in Cicchetti D. (Ed.), Developmental Psychopathology, New York, J. Wiley, https://doi.org/10.1002/9781119125556.devpsy406.

Masterson V.A., Stedman R.C., Enqvist J., Tengö M., Giusti M., Wahl D., Svedin U., 2017. The contribution of sense of place to social-ecological systems research: a review and research agenda, Ecology and Society, 22, 1, 49, https://doi. org/10.5751/ES-08872-220149.

Mathieu N., 2016. Modes d'habiter, in Choné A., Hajek I., Hamman P. (Eds), Guide des humanités environnementales, Villeneuve-d'Ascq, Presses universitaires du Septentrion, 567-582.

Michel-Guillou E., Krien N., Meur-Ferec C., 2016. Inhabitants of coastal municipalities facing coastal risks: understanding the desire to stay, Papers on social representations, London School of Economics and Political Science, 2521, 8-9.

Moles A., 1995. Psycho-géographie, in Bailly A.S., Ferras R., Pumain D. (Eds), Encyclopédie de la géographie, Paris, Economica, 177-205.

Moscovici S., 1976. Social influence and social change, London/New York, Academic Press.

Paquot T., Lussault M., Younès C. (Eds), 2007. Habiter, le propre de l'humain. Villes, territoires et philosophie, Paris, La Découverte.

Peretti-Watel P., 2003. Sociologie du risque, Paris, Armand Colin.

Pinchemel P., Pinchemel G., 1997. La face de la terre. Éléments de géographie, Paris, Armand Colin.

Proshansky H.M., 1978. The city and self-identity, Environment and Behavior, 10, 2, 147-169, https://doi.org/10.1177/ 0013916578102002.

Proshansky H.M., Fabian A.K., Kaminoff R., 1983. Placeidentity: physical world socialization of the self, Journal of Environmental Psychology, 3, 1, 57-83, https://doi.org/ 10.1016/S0272-4944(83)80021-8.
Quinn T., Bousquet F., Guerbois C., Sougrati E., Tabutaud M., 2018. The dynamic relationship between sense of place and risk perception in landscapes of mobility, Ecology and Society, 23, 2, https://doi.org/10.5751/ES-10004-230239.

Quinn T., Bousquet F., Guerbois C., Heider L., Brown K., 2019. How local water and waterbody meanings shape flood risk perception and risk management preferences, Sustainability Science, 14, 565-578, https://doi.org/ 10.1007/s11625-019-00665-0.

Radkowski G.-H. de, 2002. Anthropologie de l'habiter. Vers le nomadisme, Paris, Presses universitaires de France.

Rey-Valette H., Rulleau B., Hellequin A.-P., Meur-Ferec C., Flanquart H., 2015. Second-home owners and sea-level rise: the case of the Languedoc-Roussillon region (France), Journal of Policy Research in Tourism, Leisure and Events, 7, 1, 32-47, https://doi.org/10.1080/19407963.2014.942734.

Rocle N., Bouet B., Chasseriaud S., Lyser S., 2016. Tant qu'il y aura des « profanes »... dans la gestion des risques littoraux. Le cas de l'érosion marine à Lacanau, VertigO La revue électronique en sciences de l'environnement, 16, 2, https:// doi.org/10.4000/vertigo.17646.

Seamon D., Sowers J., 2008. Place and Placeness (1976): Edward Relph, in Hubbard P., Kitichin R., Valentine G. (Eds), Key texts in human geography, London, Sage, 43-52, https://www.doi.org/10.4135/9781446213742.n6.

Schultz P.W., 2001. The structure of environmental concern: concern for self, other people, and the biosphere, Journal of Environmental Psychology, 21, 4, 327-339, https://doi.org/ 10.1006/jevp.2001.0227.

Sébastien L., 2016. L'attachement au lieu, vecteur de mobilisation collective? Étude de cinq territoires ruraux, Norois, 238-239, 1, 23-41, https://doi.org/10.4000/norois.5846.

Stedman R.C., 2002. Toward a social psychology of place: predicting behavior from place-based cognitions, attitude, and identity, Environment and Behavior, 34, 5, 561-581, https://doi.org/10.1177/0013916502034005001.

Stedman R.C., 2003. Is it really just a social construction? The contribution of the physical environment to sense of place, Society \& Natural Resources, 16, 8, 671-685, https://doi. org/10.1080/08941920309189.

Stern P.C., 2000. Psychology and the science of humanenvironment interactions, American psychologist, 55, 5, 523-530, https://doi.org/10.1037/0003-066X.55.5.523.

Stern P.C., Dietz T., 1994. The value basis of environmental concern, Social Issues, 50, 3, 65-84, https://doi.org/ 10.1111/j.1540-4560.1994.tb02420.x.

Stern P.C., Dietz T., Guagnano G.A., 1995. The new ecological paradigm in social-psychological context, Environment and Behavior, 27, 6, 723-743, https://doi.org/10.1177/ 0013916595276001.

Stock M., 2006. L'hypothèse de l'habiter poly-topique: pratiquer les lieux géographiques dans les sociétés à individus mobiles, EspacesTemps.net, www.espacestemps. net/articles/hypothese-habiter-polytopique/.

Stock M., 2007. Théorie de l'habiter. Questionnements, in Paquot T., Lussault M., Younès C. (Eds), Habiter, le propre de l'humain. Villes, territoires et philosophie, Paris, La Découverte, 103-125. 
Stokols D., Shumaker S.A., 1981. People in places: a transactional view of settings, in Harvey J.H. (Ed.), Cognition, social behavior, and the environment, Hillsdale, L. Erlbaum, 441-488.

Tuan Y.-F., 1961. Topophilia, Landscape, 11, 1, 29-32.

Twigger-Ross C.L., Uzzell D.L., 1996. Place and identity processes, Journal of Environmental Psychology, 16, 3, 205-220, https://doi.org/10.1006/jevp.1996.0017.

Uzzell D., Pol E., Badenas D., 2002. Place identification, social cohesion, and environmental sustainability, Environment and Behavior, 34, 1, 26-53, https://doi.org/10.1177/ 0013916502034001003.

Vinet F., 2007. Approche institutionnelle et contraintes locales de la gestion du risque. Recherches sur le risque inondation en Languedoc-Roussillon. Mémoire d'habilitation à diriger des recherches, Montpellier, Université Paul Valéry-Montpellier-III (version disponible sur Hal, https:// tel.archives-ouvertes.fr/tel-00288137).
Vorkinn M., Riese H., 2001. Environmental concern in a local context: the significance of place attachment, Environment and Behavior, 33, 2, 249-263, https://doi.org/10.1177/ 00139160121972972.

Weiss K., Colbeau-Justin L., Marchand D., 2006. Entre connaissance, mémoire et oublis : représentations de l'environnement et réactions face à une catastrophe naturelle, in Weiss K., Marchand D., Psychologie sociale de l'environnement, Rennes, Presses universitaires de Rennes, 145-156.

Wester-Herber M., 2004. Underlying concerns in land-use conflicts. The role of place-identity in risk perception, Environmental Science \& Policy, 7, 2, 109-116, https://doi. org/10.1016/j.envsci.2003.12.001.

Willox A.C., Harper S.L., Ford J.D., Landman K., Houle K., Edge V.L., 2012. "From this place and of this place:" climate change, sense of place, and health in Nunatsiavut, Canada, Social Science \& Medicine, 75, 3, 538-547, https:// doi.org/10.1016/j.socscimed.2012.03.043.

Citation de l'article : Bousquet F., Rocle N., Rey-Valette H., Meur-Ferec C., Vye D., Lautrédou-Audouy N., Amalric M., Blanchet L., Lyser S., Blondy C., Becu N. Une exploration interdisciplinaire des liens entre relation au lieu et concernement. A propos des risques fluviaux et côtiers en France métropolitaine. Nat. Sci. Soc. 29, 2, 141-158. 